7 The ENFUMOSA cross-sectional European multicentre study of the clinical phenotype of chronic severe asthma. Eur Respir J 2003; 22: 470-477.

8 Simpson L, Mukherjee S, Cooper MN, et al. Sex differences in the association of regional fat distribution with the severity of obstructive sleep apnea. Sleep 2010; 33: 467-474.
9 Holguin F, Bleecker ER, Busse WW, et al. Obesity and asthma: an association modified by age of asthma onset. J Allergy Clin Immunol 2011; 127: 1486-1493.

\title{
EBUS-TBNA in the differential diagnosis of pulmonary artery sarcoma and thromboembolism
}

\section{To the Editors:}

We read with interest the correspondence published by PARK et al. [1] suggesting a potential role for endobronchial ultrasound-guided transbronchial needle aspiration (EBUSTBNA) to differentiate pulmonary artery sarcoma and thromboembolism.

Pulmonary artery sarcoma usually has its origin in the pulmonary arterial trunk and extends towards pulmonary branches, and is indeed sometimes difficult to differentiate from chronic thromboembolic disease. However, we believe that the authors' conclusions suggesting that EBUS-TBNA is safe in this setting are premature. Indeed, such an approach could be associated with significant complications.

Even if pulmonary artery sarcoma may mimic acute or chronic pulmonary thromboembolism, it has been reported $[2,3]$ that a careful analysis including medical history, chest computed tomography (CT), magnetic resonance imaging (MRI), pulmonary angiogram and positron emission tomography CT with ${ }^{18}$ F-2-fluoro-2-deoxy-D-glucose [4,5] may be sufficient to detect patients with pulmonary artery sarcoma. Chest CT classically shows hyperdense lesions, beaded peripheral pulmonary arteries, and contiguously soft tissue-filled pulmonary arteries and extravascular spread [2]. Moreover, MRI may be more specific for pulmonary artery sarcoma, showing enhancement with gadolinium contrast [2].

PARK et al. [1] performed EBUS-TBNA in a patient with acute pulmonary embolism, which was later confirmed by the complete reversibility of endovascular clots after 6 weeks of anticoagulation. Performing EBUS-TBNA in a patient with acute thromboembolism is certainly debatable and hazardous, and it is important to state that a diagnosis of acute thromboembolic disease is based on established imaging techniques, such as ventilation/perfusion lung scan, chest CT and pulmonary angiography [6].

Diagnosis of an endovascular tumour could be made by endovascular catheter biopsy [7]. In addition, as stated by PARK et al. [1], surgery is the treatment of choice in the management of pulmonary artery sarcoma (pneumonectomy, pulmonary artery resection/reconstruction or pulmonary endarterectomy) and chronic thromboembolic pulmonary disease (pulmonary endarterectomy) [6]. It is therefore important to highlight that surgery should be proposed to eligible patients with pulmonary artery sarcoma or chronic thromboembolic pulmonary hypertension, allowing diagnosis confirmation and management.

Finally, a large proportion of patients with proximal pulmonary artery chronic obstruction by sarcoma or thromboembolic material may present with pulmonary hypertension, a condition associated with a high risk of complication following transbronchial needle aspiration. Indeed, it has been clearly demonstrated that proximal obstruction of pulmonary arteries may be associated with hypertrophy of systemic bronchial arteries (fig. 1), increasing the risk of haemorrhage from transbronchial needle aspiration.

To conclude, we believe that physicians should be aware of the potential complications of EBUS-TBNA in patients with pulmonary artery sarcoma or pulmonary thromboembolic disease, and we consider that EBUS-TBNA should not be

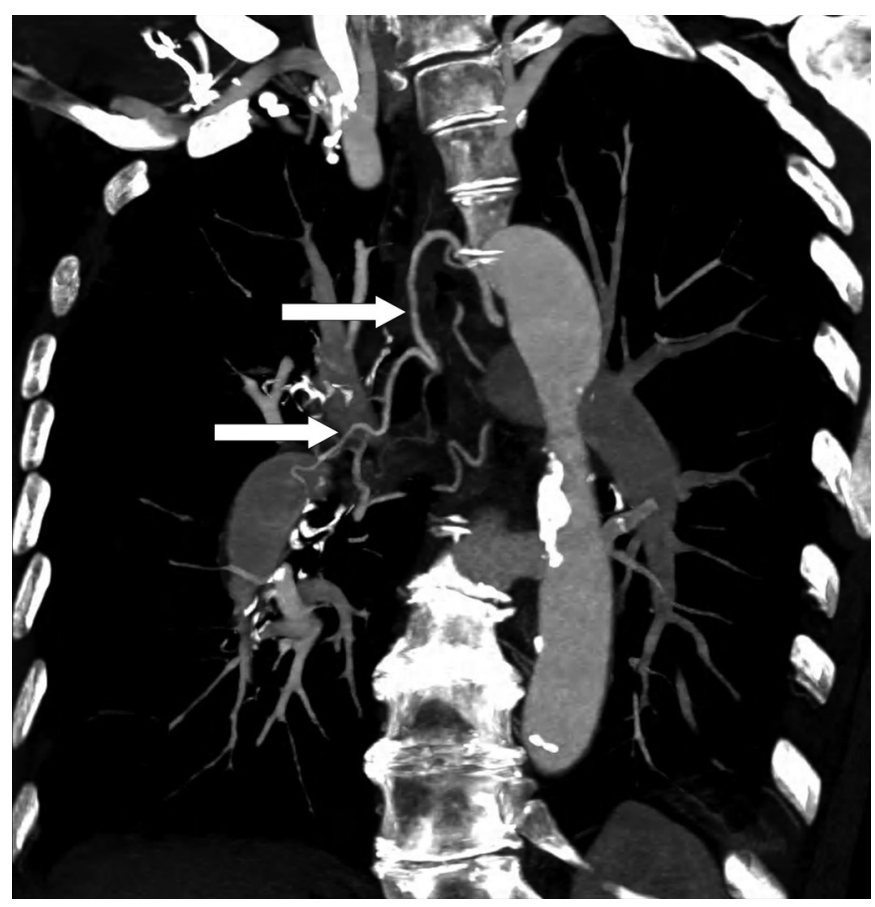

FIGURE 1. Computed tomography angiography with maximum-intensity reconstruction in the coronal plane showing hypertrophy of systemic bronchial arteries (arrows) in a case of proximal chronic thromboembolic pulmonary disease. 
proposed in the management of pulmonary artery sarcoma or thromboembolic disease.

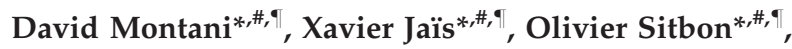 Philippe Dartevelle ${ }^{+}$, Gérald Simonneau ${ }^{*, \#, \oplus}$ and Marc Humbert $*, \#$,}

*Faculté de Médecine, Université Paris-Sud, "Assistance Publique Hôpitaux de Paris, Service de Pneumologie et Réanimation Respiratoire, Hôpital Bicêtre, Le KremlinBicêtre, "INSERM U999 LabEX LERMIT, and ${ }^{+}$Service de Chirurgie Thoracique, Centre Chirurgical Marie Lannelongue, Le Plessis-Robinson, France.

Correspondence: D. Montani, Service de Pneumologie et Réanimation Respiratoire, Hôpital Bicêtre, 78, rue du Général Leclerc, Le Kremlin-Bicêtre, 94270, France. E-mail: david.montani @abc.aphp.fr

Statement of Interest: Statements of interest for all authors of this manuscript can be found at www.erj.ersjournals.com/site/misc/ statements.xhtml

\section{REFERENCES}

1 Park JS, Chung J-H, Jheon S. EBUS-TBNA in the differential diagnosis of pulmonary artery sarcoma and thromboembolism. Eur Respir J 2011; 38: 1480-1482.

2 Blackmon SH, Rice DC, Correa AM, et al. Management of primary pulmonary artery sarcomas. Ann Thorac Surg 2009; 87: 977-984.

3 Cox JE, Chiles C, Aquino SL, et al. Pulmonary artery sarcomas: a review of clinical and radiologic features. J Comput Assist Tomogr 1997; 21: 750-755.

4 Ito K, Kubota K, Morooka M, et al. Diagnostic usefulness of ${ }^{18}$ F-FDG $\mathrm{PET} / \mathrm{CT}$ in the differentiation of pulmonary artery sarcoma and pulmonary embolism. Ann Nucl Med 2009; 23: 671-676.

5 Chong S, Kim TS, Kim BT, et al. Pulmonary artery sarcoma mimicking pulmonary thromboembolism: integrated FDG PET/ CT. AJR Am J Roentgenol 2007; 188: 1691-1693.

6 Mayer E, Jenkins D, Lindner J, et al. Surgical management and outcome of patients with chronic thromboembolic pulmonary hypertension: results from an international prospective registry. J Thorac Cardiovasc Surg 2011; 141: 702-710.

7 Winchester PA, Khilnani NM, Trost DW, et al. Endovascular catheter biopsy of a pulmonary artery sarcoma. AJR Am J Roentgenol 1996; 167: $657-659$

\section{Nomogram to predict the presence of EGFR activating mutation in lung adenocarcinoma}

\section{To the Editors:}

GIRARD et al. [1] developed a nomogram to predict the presence of epidermal growth factor receptor (EGFR) activating mutations. Before using it in practice, however, the precision and limitations must be considered. Patient selection for tyrosine kinase inhibitors (TKIs) based exclusively on EGFR mutations is still controversial, because clinical response rates to TKI are nearly $70 \%$ in tumours harbouring EGFR activating mutations. Other factors, such as amplification of the EGFR gene and the activity of molecules downstream of EGFR, such as phosphorylated protein kinase B, and mutations of KRAS can play a role in the sensitivity to EGFR inhibitors. Again, $10-20 \%$ of patients with a partial response to gefitinib did not have identifiable EGFR mutations, signifying that EGFR mutations are not the only determinants of TKI response [2]. The incidence of EGFR mutations in East Asia is nearly $40 \%$, compared with $10 \%$ in Europe and the USA. The prediction accuracy of the nomogram for EGFR mutation is unsatisfactory as the concordance index is low (0.64) in the East-Asian population, where the need for reliable predictive nomograms is higher [1].

Differences in all the above factors and population characteristics can challenge the accuracy and concordance of nomograms when they are applied to an Asian/non-Asian population. Before a nomograms is put to widespread use, it needs external independent validation. Despite external validitation, we do not know whether the existing evidence supports the impact of nomograms on medical decision making. There are no randomised studies that clearly reveal that the use of prediction nomograms improves decision making or patient care, reduces patient anxiety and is harmless.

In Chinese nonsmall cell lung cancer patients, the EGFR mutation status determined in serum DNA using mutantenriched sequencing corresponds to that demonstrated in paired tumour tissues (concordance rate of 93.1\%) suggesting that serum DNA is a practical and reliable source of tumour DNA for detecting EGFR mutations [3]. This concordance rate (93.1) is higher than the nomogram's concordance index of 0.84 and 0.64 in non-Asian and East-Asian populations, respectively. Another study confirmed high sensitivity (92\%) and specificity $(100 \%)$ of plasma EGFR mutation analysis by microfluidics digital PCR [4]. If we are unable to obtain tumour tissue or block, where diagnosis is based only on needle aspiration or cytology, it is practicable now to use serum DNA to detect EGFR mutation status and to evaluate its potential as a predictor of response to EGFR-TKIs without waiting for biopsy tissue.

In this scenario, nomograms can be built to predict the treatment outcome of TKIs. Predictive models can help 